\title{
Possible links between the liquid-gas and deconfinement-hadronization phase transitions
}

\author{
I.N. Mishustin ${ }^{1,2}$ \\ ${ }^{1}$ Frankfurt Institute for Advanced Studies, \\ J.W. Goethe Universität, Max-von-Laue Str. 1, \\ D-60438 Frankfurt am Main, Germany \\ ${ }^{2}$ Kurchatov Institute, Russian Research Center, \\ Kurchatov Sq. 1, 123182 Moscow, Russia
}

\begin{abstract}
It is commonly accepted that strongly interacting matter has several phase transitions in different domains of temperature and baryon density. In this contribution I discuss two most popular phase transitions which in principle can be accessed in nuclear collisions. One of them, the liquid-gas phase transition, is well established theoretically and studied experimentally in nuclear multifragmentation reactions at intermediate energies. The other one, the deconfinement-hadronization phase transition, is at the focus of present and future experimental studies with relativistic heavyion beams at SPS, RHIC and LHC. Possible links between these two phase transitions are identified from the viewpoint of their manifestation in violent nuclear collisions.
\end{abstract}




\section{GENERAL REMARKS}

A primary goal of present and future experiments on heavy-ion collisions is to study properties of strongly interacting matter away from the nuclear ground state. Main efforts are focused on searching for possible phase transitions in such collisions. Several phase transitions are predicted in different domains of temperature $(T)$-baryon density $\left(\rho_{B}\right)$ plane. As is well known, strongly interacting matter has at least one multi-baryon bound state at $\rho_{B}=\rho_{0} \approx 0.16 \mathrm{fm}^{-3}$ and a binding energy of about $10 \mathrm{MeV}$, corresponding to atomic nuclei, which can be considered as droplets of nuclear matter. This means that the equation of state of symmetric nuclear matter has a zero-pressure point at $\rho_{B}=\rho_{0}$. Since the pressure should also vanish at $\rho_{B} \rightarrow 0$, it must be a non-monotonic function of $\rho_{B}$, i. e. $\partial P / \partial \rho_{B}<0$ in a certain temperature-density domain. This condition signals instability of matter with respect to growing density fluctuations, a characteristic feature of the liquid-gas phase transition. Therefore, it follows from the very existence of the nuclear bound state that there should be a first order phase transition of the liquid-gas type in normal nuclear matter at subsaturation densities, $\rho_{B}<\rho_{0}$, and low temperatures, $T \leq 10 \mathrm{MeV}$.

The nuclear liquid-gas phase transition manifests itself most clearly in a nuclear multifragmentation phenomenon, observed in intermediate-energy nuclear reactions. Here we mention only a few guiding ideas which helped to identify this phase transition. The first one is the anomaly (plateau) in the caloric curve, which was first predicted theoretically 1]

and later found experimentally [2]. More recently, an interesting proposal was made [3, 4] to look for anomalous energy fluctuations in the multifragmentation events, which might be a good signal of a first order phase transition in finite systems. Another productive idea proposed in ref. [5] was to search for residual signals of the spinodal decomposition expected in connection with a liquid-gas phase transition. Such a signal, although small, was indeed found experimentally as an enhanced emission of equal-size fragments [6]. Other evidences for the liquid-gas phase transition include large fluctuations in the partition space, bimodality [7, 8], or critical behavior near the critical point [8, 9, 10].

The situation at high $T$ and non-zero baryon chemical potential $\mu_{B}$ is not so clear, although it is expected that the deconfinement and chiral transitions occur at high enough $T$ and $\rho_{B}$. As the result, a new state of matter, the Quark-Gluon Plasma (QGP), should be formed. A rigorous theoretical background for these studies is provided by the QCD 
based numerical simulations on a lattice. However, at present reliable lattice calculations exist only for $\mu=0$, i.e. $\rho_{B}=0$, where they predict a smooth deconfinement transition (crossover) at $T \approx 170 \mathrm{MeV}[11]$. As model calculations show, the QCD phase diagram in the $\left(T, \mu_{B}\right)$ plane may contain a first-order transition line (below called the critical line) which ends at a (tri)critical point [12, 13, 14]. Unfortunately, at finite $\mu$ the lattice calculations suffer from the so called "sign problem" and cannot be done easily. Different approximation schemes lead to differing predictions concerning the existence of a critical point (see e.g. refs. 15, 16, 17]). Possible signatures of this point in heavy-ion collisions were discussed in ref. [18]. However, it is unclear at present whether critical fluctuations associated with the second-order phase transition can develop in a rapidly expanding system produced in a relativistic heavy-ion collision, because of the critical slowing down effect [19]. A more promising strategy would be to search for a first-order phase transition, which may have more spectacular manifestations, as we discuss below.

Relative to the liquid-gas transition, the exploration of the QCD phase diagram is considerably more challenging. On the theoretical side, we have no tractable models to predict how the phase diagram looks in the $T-\mu$ plane, nor where the dynamical trajectories of expanding matter go. Since, by the nature of a phase transition, the effective degrees of freedom are different in the two phases, often two different models are applied below and above the critical line. Moreover, lattice QCD can only be applied to systems in statistical equilibrium, i. e. it cannot be used for dynamical simulations in real time. With regard to dynamical models, the best candidate is perhaps fluid dynamics which needs no specific information about the structure of the matter but merely macroscopic quantities such as the equation of state and kinetic coefficients. However, in its standard form this model is unsuitable for studies of unstable regimes associated with a first-order phase transition. Thus, it is very difficult to provide experimentalists with quantitative guidance to ensure that the parameters of the experiments are those where the phase transition signals are best seen.

On the experimental side, the exploration of the QCD phase structure is made extra complicated by the fact that only the hadronic phase survives in the final state [42], in contrast to the nuclear liquid-gas transition where both phases can occur in the final state. Therefore, the experience accumulated in the liquid-gas phase transition studies may be very useful for designing the analysis techniques for the exploration of the deconfinement-hadronization 
phase transition.

A similarity between the liquid-gas phase transition and the deconfinement - hadronization transition is the presence of more than one conserved charge: at low energy we have electric charge (Z) and mass number (A), while at high energy, in addition to baryon number $\mathrm{B}$ (which is identical to A) and electric charge $\mathrm{Q}$ (which corresponds to $\mathrm{Z}$ ), we have also strangeness $(\mathrm{S})$. Therefore, the lessons learned at low energy regarding multicomponent systems, in particular the isospin degree of freedom, may be helpful for the QGP studies, too.

Finally, notwithstanding the large uncertainty with regard to the value of the critical baryon density (above which the deconfinement transition is first order), it appears likely that the first-order transition can best be studied experimentally in the region of moderate bombarding energies where compressed matter is characterized by a considerable net baryon density. As we know now, a strongly interacting matter produced at RHIC, presumably a hot quark-gluon plasma, has practically vanishing net baryon density [21]. While more suitable conditions may well have been achieved already at SPS, those data have not been analyzed in a way which would unambiguously demonstrate the QGP formation. To study the first-order transition of the QCD phase diagram, the most promising facility for the future is the planned FAIR at GSI, where compressed baryonic matter is one of the prime areas of intended research.

A striking feature of central heavy-ion collisions at high energies, confirmed in many experiments (see e.g. [22, 23]), is a very strong collective expansion of matter at later stages of the reaction. This process looks like an explosion with the matter flow velocities comparable with the speed of light. The applicability of equilibrium concepts for describing phase transitions under such conditions becomes questionable and one should expect strong non-equilibrium effects. Below we demonstrate that non-equilibrium phase transitions in rapidly expanding matter can lead to interesting phenomena which, in a certain sense, are even easier to observe. 


\section{DYNAMICAL FRAGMENTATION OF A METASTABLE PHASE}

\section{A. Nuclear liquid-gas transition}

Let us consider a simple model showing how the collective flow can modify the conventional picture of a first-order phase transition [24]. Let us consider first the liquid-gas transition in nuclear matter. We assume that a system expands uniformly with the collective velocity field of a Hubble type, $\mathbf{v}_{f}(\mathbf{r})=H \mathbf{r}$, where $H$ is an appropriate Hubble constant. The expansion acts against the attractive forces which keep the nucleons together at normal density. Therefore, instead of uniformly expanding the whole system, it is energetically more favorable to split it into droplets which preserve a sufficiently high density inside, to keep attractive forces acting, and recede from each other according to the Hubble law. The space between the droplets is almost empty so that the energy cost for producing such an inhomogeneous state may be estimated as an extra interface area times a surface tension coefficient $\sigma$. One should expect that in violent reactions where thermal excitation is high, $\sigma$ might be significantly reduced compared to the value of about $1 \mathrm{MeV} / \mathrm{fm}^{2} \mathrm{known}$ for cold nuclei. The shape of the droplets, which is determined by the local density fluctuations, might be also quite complicated. But for our order-of-magnitude estimates we assume that the system splits into more or less spherical droplets of a similar size.

Now let us imagine that at the stage of the break-up the expanding system is represented by the collection of droplets with density $\rho_{B} \approx \rho_{0}$ (nuclear fragments) separated by fully developed surfaces. Within the leptodermous approximation the total energy of an individual spherical droplet of radius $R=\left(3 A / 4 \pi \rho_{B}\right)^{1 / 3}$ can be decomposed as

$$
E=E_{\mathrm{bulk}}+E_{\mathrm{kin}}+E_{\mathrm{sur}}
$$

Here the bulk term at $\rho_{B} \neq \rho_{0}$ can be written as

$$
E_{\mathrm{bulk}}=\left[a_{V}+\frac{K}{2}\left(1-\frac{\rho_{B}}{\rho_{0}}\right)^{2}\right] \cdot A,
$$

where $a_{V}$ is the bulk coefficient in the Weizsäcker formula and $K$ is the incompressibility modulus. The kinetic energy of an individual droplet, associated with its collective expansion with respect to the center of mass, is easily calculated,

$$
E_{\mathrm{kin}}=\int_{0}^{R} \frac{1}{2} m_{N} v_{f}^{2}(r) \rho(r) 4 \pi r^{2} d r=\frac{2 \pi}{5} m_{N} H^{2} \rho_{B} R^{5},
$$


where $m_{N}$ is the nucleon mass. The surface energy of a droplet is $4 \pi R^{2} \sigma$. It is worth noting that the collective kinetic energy acts here as an effective long-range potential similar to the Coulomb potential in nuclei.

To find the optimal droplet size one can apply Grady's argument [25] that the redistribution of matter is a local process that minimizes the energy per droplet volume, $\Delta E / V$. Then, since the bulk contribution does not depend on $R$, the minimization condition constitutes the balance between the collective kinetic energy and interface energy. This gives for the optimal droplet mass

$$
\bar{A}=\frac{4 \pi}{3} \rho R^{3}=\frac{20 \pi}{3} \frac{\sigma}{m_{N} H^{2}}
$$

It is determined by only two parameters: the surface tension $\sigma$ and the Hubble constant $H$. The latter one can be estimated from flow observables. For instance, in central $\mathrm{Au}+\mathrm{Au}$ collisions at 150, 250 and $400 \mathrm{MeV} /$ nucleon the measured flow velocities $v_{f}$ are $0.20 \mathrm{c}, 0.26 \mathrm{c}$ and $0.34 \mathrm{c}$ respectively [22]. Now one can estimate the Hubble constant as $H^{-1}=R_{A u} / v_{f}$, which gives 35, 26 and $20 \mathrm{fm} / \mathrm{c}$, respectively. To get the mean fragment mass $\bar{A} \approx 3$, as seen in experiment, one should take in eq. (44) $\sigma \approx 0.2 \mathrm{MeV} / \mathrm{fm}^{2}$, which is about a factor 5 smaller than in cold nuclei! Maybe this is not surprising because at a "temperature" $17 \mathrm{MeV}$, obtained for this reaction, $\sigma$ would already vanish in a thermodynamically equilibrated system. One should bear in mind, however, that the observed cold fragments are produced from hot primary fragments after their de-excitation. Therefore, primary fragments produced at the break-up stage should be bigger.

One can use the minimum information principle [26, 27] to find the inclusive fragment mass distribution, $P(A)$. In principle, the information entropy should be defined in terms of microstate probabilities, $p_{i}$, as $\sum_{i} p_{i} \ln p_{i}$. Since we are interested only in the inclusive mass distribution, we can sum up all microstates containing the fragment of mass $\mathrm{A}$. Then the information function can be defined simply as $\sum_{A} P(A) \ln P(A)$. Minimizing this function under constraint that the average fragment mass is fixed, $\bar{A}=\sum_{A} A P(A)$, we get the normalized mass distribution of the form

$$
P(A)=\frac{1}{\bar{A}} \exp \left(-\frac{A}{\bar{A}}\right) .
$$

This kind of mass distribution has been seen in numerical simulations [28] as well as in the free-jet fragmentation experiments [27]. It is remarkable that exactly this type of mass 
(charge) distributions is also observed in nuclear experiments! For instance, exponential fragment charge distributions have been found in central $\mathrm{Au}+\mathrm{Au}$ collisions at 150, 250 and $400 \mathrm{MeV} /$ nucleon [22] discussed above. By applying naively the statistical approach to these reactions one obtains charge distributions which are much too steep (smaller $\bar{A}$ ).

\section{B. Deconfinement-hadronization transition}

A similar scenario can also be considered for the deconfine-ment-hadronization phase transition in relativistic nuclear collisions 29, 30]. The difference will be mainly in the parameters characterizing this phase transition. Of course, this consideration is justified only for the first-order phase transition, which is expected at moderate $\mathrm{T}$ and high enough $\rho_{B}$ (see discussion in the Introduction). Most likely, this picture does not apply for the RHIC energies, where produced matter is characterized by very high $\mathrm{T}$ and very low $\mu$ [21], corresponding to the crossover transition.

For simplicity, below we use capital letters Q and $\mathrm{H}$ (not to be confused with the Hubble constant $H$ ) for the deconfined (quark-gluon) phase and the hadronic phase, respectively. Let us assume that the dynamical fragmentation of the deconfined phase has resulted in a collection of Q droplets embedded in a dilute $\mathrm{H}$ phase, as illustrated in Fig. 1. The optimal droplet size can be determined by applying the same energy balance prescription discussed above. The only difference is that the droplet mass with respect to the hadronic background is now calculated as $M=\Delta \mathcal{E} V$, where $\Delta \mathcal{E}=\mathcal{E}_{Q}-\mathcal{E}_{H}$ is the energy density difference of $\mathrm{Q}$ and $\mathrm{H}$ bulk phases, and $V$ is the volume of the droplet. Applying Grady's minimization rule we get the optimum droplet radius

$$
R^{*}=\left(\frac{5 \sigma}{\Delta \mathcal{E} H^{2}}\right)^{1 / 3} .
$$

As eq. (6) indicates, the droplet size depends strongly on $H$. When expansion is slow (small $H$ ) the droplets are big. In the adiabatic limit the process may look like a fission of a cloud of plasma. But fast expansion should lead to very small droplets. This state of matter is very far from a thermodynamically equilibrated mixed phase, particularly because the $\mathrm{H}$ phase is very dilute. One can say that the metastable $\mathrm{Q}$ matter is torn apart by a mechanical strain associated with the collective expansion. This has a direct analogy with the dynamical multifragmentation process, described in the previous section, or with the 


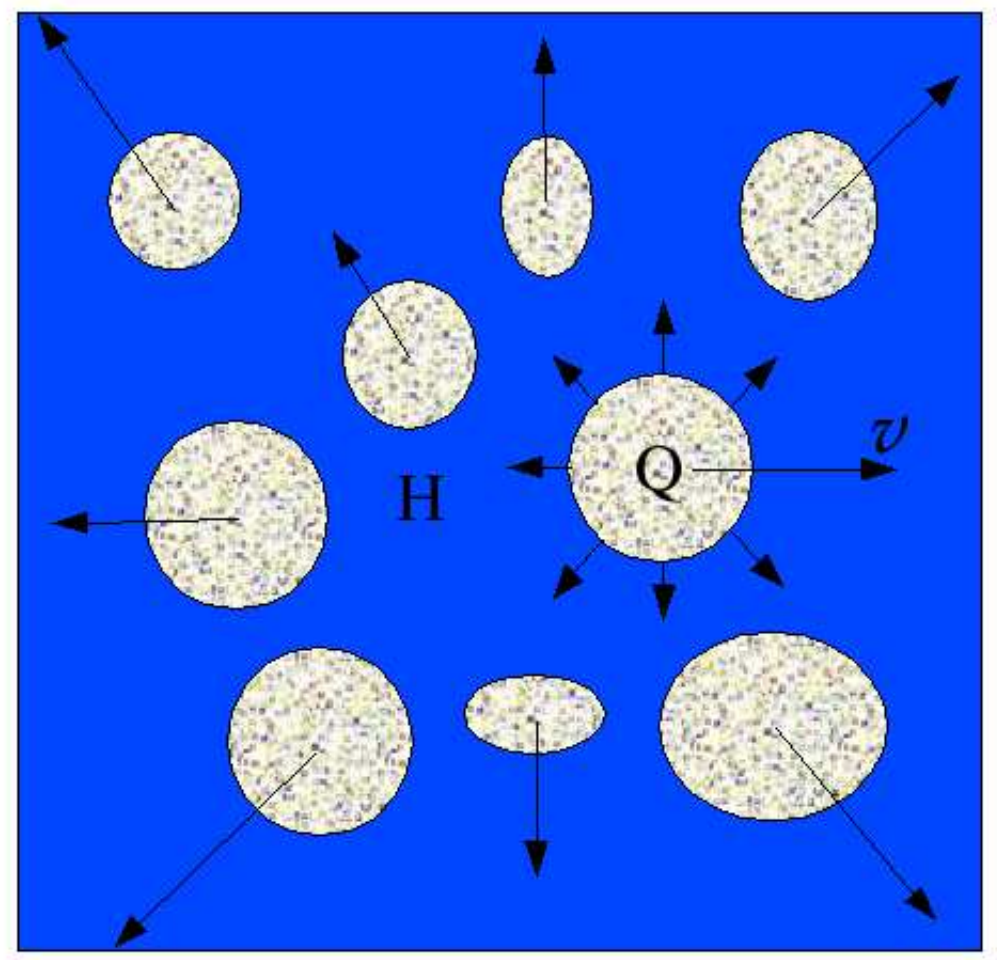

FIG. 1: Schematic view of the multi-droplet state produced after the dynamical fragmentation of a metastable high energy-density phase (in this example, the Q phase). The droplets are embedded in the low energy-density phase (in this example, the $\mathrm{H}$ phase). Each droplet expands individually as well as participates in the overall Hubble-like expansion.

fragmentation of pressurized fluids leaving nozzles [27].

The driving force for expansion is the pressure gradient, $\nabla P \equiv c_{s}^{2} \nabla \mathcal{E}$, which depends crucially on the sound velocity in matter, $c_{s}$. Here we are interested in the expansion rate of the partonic phase, which is not directly observable but predicted by the hydrodynamical simulations. In the vicinity of the phase transition, one may expect a "soft point" [31, 32] where the sound velocity is smallest and the ability of matter to generate the collective expansion is minimal. If the initial state of the $\mathrm{Q}$ phase is close to this point, its subsequent expansion will be slow. Accordingly, the droplets produced in this case will be big. When moving away from the soft point, one would see smaller and smaller droplets. For numerical 
estimates we choose two values of the Hubble constant: $H^{-1}=20 \mathrm{fm} / \mathrm{c}$ to represent the slow expansion from the soft point and $H^{-1}=6 \mathrm{fm} / \mathrm{c}$ for the fast expansion.

One should also specify two other parameters, $\sigma$ and $\Delta \mathcal{E}$. The surface tension $\sigma$ is a subject of debate at present. Lattice simulations indicate that it could be as low as a few $\mathrm{MeV} / \mathrm{fm}^{2}$ in the vicinity of the critical line. However, for our non-equilibrium scenario, more appropriate values are closer to $10-20 \mathrm{MeV} / \mathrm{fm}^{2}$, which follow from effective chiral models. As a compromise, the value $\sigma=10 \mathrm{MeV} / \mathrm{fm}^{2}$ is used below for rough estimates. Bearing in mind that nucleons and heavy mesons are the smallest droplets of the Q phase, one can take $\Delta \mathcal{E}=0.5 \mathrm{GeV} / \mathrm{fm}^{3}$, i.e. the energy density inside the nucleon. Then one gets $R^{*}=3.4 \mathrm{fm}$ for $H^{-1}=20 \mathrm{fm} / \mathrm{c}$ and $R^{*}=1.5 \mathrm{fm}$ for $H^{-1}=6 \mathrm{fm} / \mathrm{c}$. As follows from eq. (6), for a spherical droplet $V \propto 1 / \Delta \mathcal{E}$, and in the first approximation its mass,

$$
M^{*} \approx \Delta \mathcal{E} V=\frac{20 \pi}{3} \frac{\sigma}{H^{2}}
$$

is independent of $\Delta \mathcal{E}$ (compare with eq. (44)). For the two values of $R^{*}$ given above the optimal droplet mass is $\sim 100 \mathrm{GeV}$ and $\sim 10 \mathrm{GeV}$, respectively. As mentioned in the previous section, the distribution of droplet masses should follow an exponential law, $\exp \left(-\frac{M}{M^{*}}\right)$. Thus, about $2 / 3$ of droplets have masses smaller than $M^{*}$, but with $1 \%$ probability one can find droplets as heavy as $5 M^{*}$.

\section{OBSERVABLE MANIFESTATIONS OF QUARK DROPLETS}

After separation, the QGP droplets will recede from each other according to the global collective expansion, predominantly in the beam direction. Therefore, their c.m. rapidities $\mathrm{y}_{i}$ will be in one-to-one correspondence with their spatial positions. One may expect that they will be distributed more or less uniformly between the target and the projectile rapidities. Since rescatterings in the dilute $\mathrm{H}$ phase are rare, most hadrons produced from individual droplets will go directly into detectors. This may explain why freeze-out parameters extracted from the hadronic yields are close to the phase transition boundary [21]. Indeed, due to the rapid expansion it is unlikely that the thermodynamical equilibrium will be established between the $\mathrm{Q}$ and $\mathrm{H}$ phases or within the $\mathrm{H}$ phase alone. If this were to happen, the final $\mathrm{H}$ phase would be more or less uniform, and thus, no traces of the droplet phase would appear in the final state. 


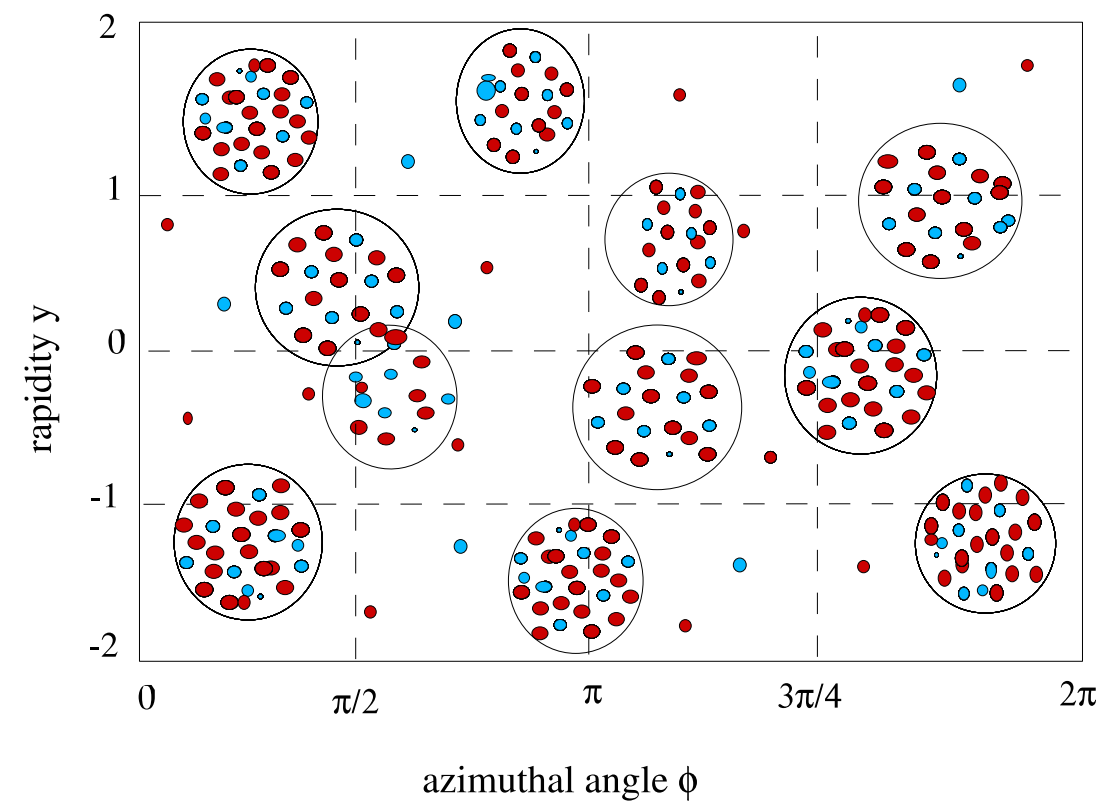

FIG. 2: Schematic view of the momentum space distribution of secondary hadrons produced from an ensemble of droplets. Each droplet emits hadrons (mostly pions) within a rapidity interval $\delta y \sim 1$ and azimuthal angle spreading of $\delta \phi \sim 1$.

The final fate of individual droplets depends on their sizes and on details of the equation of state. Due to the negative Laplace pressure, $2 \sigma / R$, the residual expansion of individual droplets will slow down. The smaller droplets may even reverse their expansion and cooling to shrinking and reheating. Then, the conversion of $\mathrm{Q}$ matter into $\mathrm{H}$ phase may proceed through the formation of the imploding deflagration front [32, 33]. Bigger droplets may expand further until they enter the region of spinodal instability At this stage the difference between 1-st and 2-nd order phase transitions or a crossover is insignificant. Since the characteristic "rolling down" time is rather short, $\sim 1 \mathrm{fm} / \mathrm{c} 34]$, the Q droplets will be rapidly converted into the non-equilibrium $H$ phase. In refs. [35, 36, 37] the evolution of individual droplets was studied numerically within a hydrodynamical approach including dynamical chiral fields. It has been demonstrated that the energy released at the spinodal decomposition can be transferred directly into the collective oscillations of the $(\sigma, \pi)$ fields which give rise to the soft pion radiation. One can also expect the formation of Disoriented Chiral Condensates (DCC) [38] in the voids between the droplets. 
It is interesting to note that the surface tension has stabilizing effect on the droplet evolution. Since the droplets are hot, their life time will be mainly determined by the rate of hadron evaporation from the surface (see also the discussion in ref. [39]). This will lead to their cooling and shrinking. One can speculate about all kinds of exotic objects, like e.g. strangelets, glueballs, formed in this way. The possibility of forming "vacuum bubbles', i.e. regions with depleted quark and gluon condensates, was discussed in ref. [35]. All these interesting possibilities deserve further study and numerical simulations.

In the droplet phase the mean number of produced hadrons in a given rapidity interval is

$$
\langle N\rangle=\sum_{i}^{N_{D}} \overline{n_{i}}=\langle n\rangle\left\langle N_{D}\right\rangle,
$$

where $\overline{n_{i}}$ is the mean multiplicity of hadrons emitted from a droplet i, $\langle n\rangle$ is the average multiplicity per droplet and $\left\langle N_{D}\right\rangle$ is the mean number of droplets produced in this interval. If droplets do not overlap in rapidity space, each of them will give a bump in the hadron rapidity distribution around its center-of-mass rapidity $y_{i}[29$, 34]. In case of a Boltzmann spectrum the width of the bump will be $\delta y \approx \sqrt{T / m}$, where $T$ is the droplet temperature and $m$ is the particle mass. At $T \sim 100 \mathrm{MeV}$ this gives $\delta y \approx 0.8$ for pions and $\delta y \approx 0.3$ for nucleons. These spectra might be slightly modified by the residual expansion of droplets. Due to the radial expansion of the fireball the droplets should also be well separated in the azimuthal angle. The characteristic angular spreading of pions produced by an individual droplet is determined by the ratio of the thermal momentum of emitted pions to their mean transverse momentum, $\delta \phi \approx 3 T /\left\langle p_{\perp}\right\rangle \sim 1$. The resulting phase-space distribution of hadrons in a single event will be a superposition of contributions from different $\mathrm{Q}$ droplets superimposed on a more or less uniform background from the $\mathrm{H}$ phase. Such a distribution is shown schematically in Fig. 2. It is obvious that such inhomogeneities (clusterization) in the momentum space will be reflected in strong non-statistical fluctuations of hadron multiplicities measured in a given rapidity and angular window. The fluctuations will be more pronounced if primordial droplets are big, as expected in the vicinity of the soft point. If droplets as heavy as $100 \mathrm{GeV}$ are formed, each of them will emit up to 200 pions within a narrow rapidity and angular interval, $\delta y \sim 1, \delta \phi \sim 1$. If only a few droplets are produced on average per unit rapidity, $N_{D} \gtrsim 1$, they will be easily resolved and analyzed. On the other hand, the fluctuations will be suppressed by a factor $\sqrt{N_{D}}$ if many small droplets fall 
in the same rapidity interval.

It is convenient to characterize the multiplicity fluctuations in a given rapidity window by the scaled variance

$$
\omega_{N} \equiv \frac{\left\langle N^{2}\right\rangle-\langle N\rangle^{2}}{\langle N\rangle}
$$

Its important property is that $\omega_{N}=1$ for the Poisson distribution, and therefore any deviation from unity will signal a non-statistical emission mechanism. As shown in ref. [40], for an ensemble of emitting sources (droplets) $\omega_{N}$ can be expressed in a simple form, $\omega_{N}=$ $\omega_{n}+\langle n\rangle \omega_{D}$, where $\omega_{n}$ is an average multiplicity fluctuation in a single droplet, $\omega_{D}$ is the fluctuation in the droplet mass distribution and $\langle n\rangle$ is the mean multiplicity from a single

droplet. Since $\omega_{n}$ and $\omega_{D}$ are typically of order of unity, the fluctuations from the multidroplet emission are enhanced by the factor $\langle n\rangle$. According to the picture of a first-order phase transition advocated above, this enhancement factor can be as large as $10 \div 100$. A more detailed consideration of the multiplicity distributions associated with the hadron emission from an ensemble of droplets is given in ref. 30]. Until now no strong anomalies in hadron multiplicity distributions have been observed in relativistic heavy-ion collisions (see e. g. ref. [41]).

\section{CONCLUSIONS}

- It is most likely that strongly interacting matter has at least two first-order phase transitions, i.e. the nuclear liquid-gas transition and the deconfinement-hadronization transition. Their unambiguous experimental identification is the main goal of heavy-ion collision experiments at present and future facilities. Studying phase transitions in such a dynamical environment should take into account strong non-equilibrium effects.

- A first-order phase transition in rapidly expanding matter should proceed through the nonequilibrium stage when a metastable phase splits into droplets whose size is inversely proportional to the expansion rate. The primordial droplets should be biggest in the vicinity of a soft point when the expansion is slowest.

- Hadron emission from droplets of the quark-gluon plasma should lead to large nonstatistical fluctuations in their rapidity and azimuthal spectra, as well as in multiplicity distributions in a given rapidity window. The hadron abundances may reflect directly the chemical composition in the plasma phase. 
- To identify the phase transition threshold, the measurements should be done at different collision energies. The predicted dependence on the expansion rate and the reaction geometry can be checked in collisions with different ion masses and impact parameters.

- If the first-order deconfinement/chiral phase transition is only possible at finite baryon densities, one should try to identify it by searching for the anomalous fluctuations in the regions of phase space characterized by a large baryon chemical potential. These could be the nuclear fragmentation regions in collisions with very high energies (high-energy SPS, RHIC, LHC) or the central rapidity region in less energetic collisions (AGS, low-energy SPS, future GSI facility FAIR).

- A rich experience has been accumulated in theoretical and experimental studies of nuclear multifragmentation as a signal of the liquid-gas phase transition in normal nuclear matter. These lessons may be useful in present and future studies of the deconfinementhadronization and chiral phase transitions in relativistic heavy-ion collisions.

I thank J. Randrup and F. Gulminelli for fruitful discussions and useful advises. This work was supported in part by the grants RFFR 05-02-04013 and NS-8756.2006.2 (Russia).

[1] J.P. Bondorf, R. Donangelo, I.N. Mishustin, H. Schulz, Nucl. Phys. A444, 460 (1985).

[2] J. Pochodzalla and ALADIN Collaboration, Phys. Rev. Lett. 75, 1040 (1995).

[3] Ph. Chomaz, F. Gulminelli, and V. Duflot, Phys. Rev. E64, 046114 (2001).

[4] M. D'Agostino et al., Phys. Lett. B473, 219 (2000).

[5] Ph. Chomaz, M. Colonna and J. Randrup, Phys. Rep. 389, 263 (2004).

[6] B. Borderie et al., Phys. Rev. Lett. 86, 3252 (2001).

[7] A.S. Botvina et al., Nucl. Phys. A584, 737 (1995).

[8] M. D'Agostino et al., Nucl. Phys. A650, 329 (1999).

[9] B.K. Srivastava et al., Phys. Rev. C65, 054617 (2002).

[10] J.B. Elliott et al., Phys. Rev. Lett. 88, 042701 (2002).

[11] F. Karsch, E. Laermann, A. Peikert, Nucl. Phys. B605, 579 (2001).

[12] M.A. Halasz, A.D. Jackson, R.E. Shrock, M.A. Stephanov and J.J.M. Verbarshot, Phys. Rev. D58, 096007 (1998). 
[13] J. Berges and K. Rajagopal, Nucl. Phys. B538, 215 (1999).

[14] O. Scavenius, A. Mocsy, I.N. Mishustin, D. Rischke, Phys. Rev. C64, 045202 (2001).

[15] Z. Fodor, S.D. Katz, JHEP 0203, 014 (2002).

[16] C.R. Allton et al., Phys. Rev. D66, 074507 (2002).

[17] R.V. Gavai, S. Gupta, Phys. Rev. D68, 034506 (2003).

[18] M. Stephanov, K. Rajagopal and E. Shuryak, Phys. Rev. Lett. 81, 4816 (1998).

[19] Boris Berdnikov and Krishna Rajagopal, Phys. Rev. D61, 105017 (2000).

[20] J. Adams and STAR Collaboration, Nucl. Phys. A757, 102 (2005).

[21] P. Braun-Munzinger, D. Magestro, K. Redlich, and J. Stachel, Phys. Lett. B518, 41 (2001); P. Braun-Munzinger, J. Stachel, J. Phys. G: Nucl. Part. Phys. 28, 1971 (2002); A. Andronic, P. Braun-Munzinger, J. Stachel, Nucl. Phys. A772, 167 (2006).

[22] W. Reisdorf and FOPI Collaboration, Nucl. Phys. A612, 493 (1997).

[23] Nu Xu, Prog. Part. Nucl. Phys. 53, 165 (2004).

[24] I.N. Mishustin, Nucl. Phys. A630, 111c (1998).

[25] D.E. Grady, J. Appl. Phys. 53(1), 322 (1981).

[26] J. Aichelin, J. Hüfner, Phys. Lett. B136, 5 (1984).

[27] E.L. Knuth, U.Henne, J. Chem. Phys. 110, 2664 (1999).

[28] B.L. Holian and D.E. Grady, Phys. Rev. Lett. 60, 1355 (1988).

[29] I.N. Mishustin, Phys. Rev. Lett. 82, 4779 (1999).

[30] I.N. Mishustin, in Proc. Int. Conf. "New Trends in High-Energy Physics" (Crimea, Ukraine, September 10-17, 2005); hep-ph/0512366.

[31] C.M. Huang and E.V. Shuryak, Phys. Rev. Lett. 75, 4003 (1995); Phys. Rev. C57, 1891 (1998).

[32] D. Rischke, M. Gyulassy, Nucl. Phys. A597, 701 (1996); A608, 479 (1996).

[33] S. Digal, A.M. Srivastava, Phys. Rev. Lett. 80, 1841 (1998).

[34] L.P. Csernai, I.N. Mishustin, Phys. Rev. Lett. 74, 5005 (1995).

[35] I.N. Mishustin, O. Scavenius, Phys. Rev. Lett. 83, 3134 (1999).

[36] O. Scavenius, A. Dumitru, E. S. Fraga, J. T. Lenaghan and A. D. Jackson, Phys. Rev. D 63, 116003 (2001).

[37] K. Paech, H. Stoecker and A. Dumitru, Phys. Rev. C 68, 044907 (2003).

[38] J.D. Bjorken, K.L. Kowalski, C.C. Taylor, SLAC-PUB-6413, Sep. 1993; hep-ph/9309235. 
[39] M. Alford, K. Rajagopal and F. Wilczek, Phys. Lett. B422, 247 (1998).

[40] G. Baym, H. Heiselberg, Phys. Lett. B469, 7 (1999).

[41] M. Rybczynski and NA49 Collaboration, J. Phys. Conf. Ser. 5, 74 (2005).

[42] Some information about the deconfined phase can be obtained from the electromagnetic probes and from quenching of hard partonic jets, see e.g. ref. [20]. 